\title{
Superoxiddismutasemimetikum (GC4419) vs. Placebo zur Reduktion von schweren oralen Mukositiden infolge simultaner Radiochemotherapie mit Cisplatin bei Kopf-Hals-Tumoren: randomisierte, doppelblinde Phase-Ilb-Studie
}

\author{
Irenäus A. Adamietz' \\ Online publiziert: 19. Juni 2020 \\ (c) Der/die Autor(en) 2020
}

Hintergrund Die orale Mukositis (OM) ist eine häufige und stark beeinträchtigende Nebenwirkung der Strahlentherapie (RT) bei Kopf- und Halstumoren, die fast immer eine Intervention im Rahmen von Supportivmaßnahmen erfordert. Das Ziel dieser multiinstitutionellen, randomisierten, doppelblinden Phase-IIb-Studie war es, die Wirksamkeit und Sicherheit von GC4419, eines Superoxiddismutasemimetikums zur Reduktion oraler Mukositis, gegenüber einem Placebo zu eruieren [1].

Patientengut und Methoden In die multizentrische, prospektive Studie wurden insgesamt 223 Patienten aus 44 Institutionen in den USA und Kanada mit einem lokal fortgeschrittenen Mundhöhlen-/Oropharynxkarzinom eingeschlossen. Alle Patienten wurden einer definitiven oder postoperativen Radiochemotherapie (RCT) unterzogen. Die Gesamtstrahlendosis am Oropharynx betrug 60-72 Gy (minimale Dosis an mindestens zwei Lokalisationen der oralen Schleimhaut $>50$ Gy). Als Technik kam bei allen Patienten eine IMRT zur Anwendung. Simultan wurde Cisplatin (wöchentlich oder alle 3 Wochen) verabreicht. Alle Patienten erhielten vor jeder IMRT-Fraktion entweder GC4419 intravenös als 60-minütige Infusion oder ein Placebo. Randomisiert wurde zwischen einer Dosierung von $30 \mathrm{mg}(n=73)$ oder $90 \mathrm{mg}(n=76)$ sowie Placebo $(n=74)$. Der WHO-Grad der OM wurde während der Behandlung

Originalpublikation Anderson CM, Lee CM, Saunders DP et al (2019) Phase IIb, randomized, double-blind trial of GC4419 versus placebo to reduce severe oral mucositis due to concurrent radiotherapy and cisplatin for head and neck cancer. J Clin Oncol 37(34):3256-3265. https://doi.org/10.1200/JCO.19.01507

Prof. Dr. med. Irenäus A. Adamietz irenaeus.adamietz@elisabethgruppe.de

1 Klinik für Strahlentherapie und Radio-Onkologie, Marien Hospital Herne Universitätsklinik, Ruhr-Universität Bochum, Hölkeskampring 40, 44625 Herne, Deutschland zweiwöchentlich und bis zu 8 Wochen nach Beendigung der Therapie alle 14 Tage evaluiert. Der primäre Endpunkt war die Dauer der schweren OM, die für jede aktive Dosisstufe gegenüber dem Placebo getestet wurde (zweiseitige Intention-to-treat-Analyse bei $p=0,05)$. Für die Klassifizierung von unerwünschten Ereignissen wurde die Version 4.03 der Common Terminology Criteria for Adverse Events (CTCAE) des National Cancer Institute verwendet. Patientenund Tumormerkmale sowie die Therapiecharakteristika waren in allen Behandlungsgruppen vergleichbar. Als primäre und sekundäre abhängige Variable wurden Dauer, Inzidenz und Schweregrad der OM statistisch evaluiert.

Ergebnisse Die höhere Dosierung von GC4419 (90mg) führte im Vergleich zu Placebo zu einer signifikanten Reduktion der schweren oralen Mukositis ( $p=0,024$; Median 1,5 vs. 19 Tage). Die Inzidenz der schweren oralen Mukositis $(43 \%$ vs. $65 \% ; p=0,009)$ und der Schweregrad (Grad4-Inzidenz, $16 \%$ vs. $30 \% ; p=0,045)$ wurden ebenfalls signifikant verbessert. Die 30-mg-Dosierung zeigte Werte, die zwischen der höheren Dosierung und Placebo lagen. Das Nebenwirkungsprofil war in allen Armen vergleichbar. Eine spezifische Toxizität von GC4419 wurde nicht festgestellt. Die 2-Jahres-Nachbeobachtung der Tumorkontrolle ist noch nicht abgeschlossen.

Schlussfolgerungen der Autoren GC4419 mit einer täglichen Dosis von $90 \mathrm{mg}$ führt zu einer signifikanten, klinisch relevanten Reduzierung der radiogenen oralen Mukositis (Dauer, Inzidenz und Schweregrad). Das Nebenwirkungsprofil dieser Medikation ist akzeptabel. Eine nachfolgende Phase-III-Studie (ROMAN) hat begonnen [2]. 


\section{Kommentar}

Behandlung der radiogenen Mukositis mit Mimetika der Superoxiddismutase (SOD) - Sinn oder Unsinn? Die zweite randomisierte Studie zur Wirksamkeit von GC4419 zeigt sehr gute Ergebnisse [1]. Die vorliegenden Daten und diejenigen aus publizierten Studien haben die FDA dazu bewegt, ein beschleunigtes Zulassungsverfahren einzuleiten $[1,3,4]$. Bedeutet nun der Einsatz der SOD-Mimetika den Durchbruch bei der Prophylaxe bzw. Behandlung der radiogenen Mukositis?

Die iatrogene orale Mukositis bei Patienten mit KopfHals-Tumoren, charakterisiert durch Entzündung, Atrophie und Abbau der Schleimhaut oder der Auskleidung der Mundhöhle, gehört zur täglichen strahlentherapeutischen Erfahrung. Die damit assoziierten Schmerzen, Odynodysphagie, Dysgeusie, verminderte orale Nahrungsaufnahme und systemische Infektionen stellen alle Strahlentherapeuten vor therapeutische Herausforderungen und kompromittieren gelegentlich den onkologischen Behandlungserfolg.

Die Behandlung der radiogenen Mukositis ist multifaktoriell. Seit Langem wird diesbezüglich den radioprotektiven Substanzen ein hohes therapeutisches Potenzial zugeschrieben. Die manganhaltige Verbindung GC4419 gehört zusammen mit Amifostin, Palifermin und RRx-001 zu den Radioprotektiva mit potenziellem oder geprüftem klinischem Effekt [3].

Eine übermäßige Bildung von reaktiven Sauerstoffspezies wie Superoxid (radikales $\mathrm{O}_{2}$ ) spielt eine zentrale Rolle in der Pathogenese der Mukositis. Das antioxidative Enzym Superoxiddismutase (SOD) entgiftet Superoxide durch Umwandlung in das relativ stabile und schlecht reaktive Oxidationsmittel Wasserstoffperoxid $\left(\mathrm{H}_{2} \mathrm{O}_{2}\right)$. Dieser Effekt scheint eine klinisch messbare Reduktion der oralen Mukositis herbeiführen zu können.

Der indirekte Effekt der Strahlentherapie ist ebenfalls von der Radikalüberproduktion abhängig. Die Absorption von ionisierender Strahlung unterbricht direkt chemische Bindungen in Zellmolekülen oder verursacht Schäden durch Bildung von Radikalen, die mit nahegelegenen Molekülen reagieren und Proteine, Lipide und Nukleinsäuren schädigen. Die Summe aus Basenschäden, Zuckerschäden, Einzel- oder Doppelstrangbrüchen der DNA führt zum Zelltod [5]. Wird die Menge der Radikale reduziert, liegt die Befürchtung nahe, dass der zytotoxische Effekt am Tumor reduziert sein könnte. Die Befürworter der Substanz argumentieren jedoch, dass GC4419 Superoxid selektiv in ein $\mathrm{H}_{2} \mathrm{O}_{2}$-Molekül zerlegt, das selbst ein eigenständiges Oxidationsmittel ist und somit ebenfalls einen zytotoxischen Effekt am Tumor entfalten kann.

Strahlenbiologischen Erkenntnissen folgend, spielt die Vernichtung basaler Stammzellen der Schleimhaut eine entscheidende Rolle bei der Entstehung der radiogenen Muko- sitis. Der gleiche Mechanismus liegt der Tumorkontrolle durch Strahlentherapie zugrunde. Es wird interessant sein zu verfolgen, ob und wie die beschriebenen Wirkungen des SOD-Mimetikums zwischen den beiden Stammzellarten differenzieren können. Sollte GC4419 diese Wirkung entfalten, könnte es trotz recht aufwendiger Applikation künftig eine wichtige Rolle in der multifaktoriellen Behandlung der radiogenen Mukosaschäden einnehmen.

\section{Fazit}

Die Aufgabe des Radioonkologen ist in erster Linie die Tumorbekämpfung und erst in zweiter Linie die Kontrolle der radiogenen Mukositis. Aus diesem Grund sollte man künftig genau auf die Tumorkontrolle achten, wenn SODMimetika eingesetzt werden. Inwieweit GC4419 diesen Anforderungen gerecht wird, müssen die künftigen Studien zeigen. Sollten die Parameter der Tumorkontrolle dabei kompromittiert sein, könnte es der Substanz so ergehen wie damals Amifostin, einer Substanz, bei der ein protektiver Tumoreffekt nie sicher ausgeschlossen werden konnte.

Irenäus A. Adamietz, Herne

Funding Open Access funding provided by Projekt DEAL.

Interessenkonflikt I.A. Adamietz gibt an, dass kein Interessenkonflikt besteht.

Open Access Dieser Artikel wird unter der Creative Commons Namensnennung 4.0 International Lizenz veröffentlicht, welche die Nutzung, Vervielfältigung, Bearbeitung, Verbreitung und Wiedergabe in jeglichem Medium und Format erlaubt, sofern Sie den/die ursprünglichen Autor(en) und die Quelle ordnungsgemäß nennen, einen Link zur Creative Commons Lizenz beifügen und angeben, ob Änderungen vorgenommen wurden.

Die in diesem Artikel enthaltenen Bilder und sonstiges Drittmaterial unterliegen ebenfalls der genannten Creative Commons Lizenz, sofern sich aus der Abbildungslegende nichts anderes ergibt. Sofern das betreffende Material nicht unter der genannten Creative Commons Lizenz steht und die betreffende Handlung nicht nach gesetzlichen Vorschriften erlaubt ist, ist für die oben aufgeführten Weiterverwendungen des Materials die Einwilligung des jeweiligen Rechteinhabers einzuholen.

Weitere Details zur Lizenz entnehmen Sie bitte der Lizenzinformation auf http://creativecommons.org/licenses/by/4.0/deed.de.

\section{Literatur}

1. Anderson CM, Lee CM, Saunders DP, Curtis A, Dunlap N, Nangia C, Lee AS, Gordon SM, Kovoor P, Arevalo-Araujo R, BarAd V, Peddada A, Colvett K, Miller D, Jain AK, Wheeler J, Blakaj D, Bonomi M, Agarwala SS, Garg M, Worden F, Holmlund J, Brill JM, Downs M, Sonis ST, Katz S, Buatti JM (2019) Phase IIb, randomized, double-blind trial of GC4419 versus placebo to reduce severe oral mucositis due to concurrent radiotherapy and cisplatin 
for head and neck cancer. J Clin Oncol 37(34):3256-3265. https:// doi.org/10.1200/JCO.19.01507

2. Holmlund J, Brill JM, Lee CM, Saunders D, Sonis ST, Downs M, Anderson CM (2019) Roman: Reduction in oral mucositis with avasopasem manganese (GC4419) — Phase 3 trial in patients receiving chemoradiotherapy for locally-advanced, non-metastatic head and neck cancer. J Clin Oncol 37(15_suppl):TPS6096-TPS6096. https://doi.org/10.1200/JCO.2019.37.15_suppl.TPS6096

3. Oronsky B, Goyal S, Kim MM, Cabrales P, Lybeck M, Caroen S, Oronsky N, Burbano E, Carter C, Oronsky A (2018) A review of clinical radioprotection and chemoprotection for oral mucositis. Transl Oncol 11(3):771-778. https://doi.org/10.1016/j.tranon. 2018.03.014
4. Anderson CM, Sonis ST, Lee CM, Adkins D, Allen BG, Sun W, Agarwala SS, Venigalla ML, Chen Y, Zhen W, Mould DR, Holmlund JT, Brill JM, Buatti JM (2018) Phase 1b/2a trial of the superoxide dismutase mimetic GC4419 to reduce chemoradiotherapy-induced oral mucositis in patients with oral cavity or oropharyngeal carcinoma. Int J Radiat Oncol Biol Phys 100(2):427-435. https:// doi.org/10.1016/j.ijrobp.2017.10.019

5. Okunieff P, Swarts S, Keng P, Sun W, Wang W, Kim J, Yang S, Zhang H, Liu C, Williams JP, Huser AK, Zhang L (2008) Antioxidants reduce consequences of radiation exposure. Adv Exp Med Biol 614:165-178. https://doi.org/10.1007/978-0-387-74911-2_20 